7 Plo I, Zhang Y, Le Couédic JP, Nakatake M, Boulet JM, Itaya M et al. An activating mutation in the CSF3R gene induces a hereditary chronic neutrophilia. $J$ Exp Med 2009; 206: 1701-1707.

8 Maxson JE, Gotlib J, Pollyea DA, Fleischman AG, Agarwal A, Eide CA et al. Oncogenic CSF3R mutations in chronic neutrophilic leukemia and atypical CML. N Engl J Med 2013; 368: 1781-1790.

9 Pardanani A, Lasho TL, Laborde RR, Elliott M, Hanson CA, Knudson RA et al. CSF3R T618l is a highly prevalent and specific mutation in chronic neutrophilic leukemia. Leukemia 2013; e-pub ahead of print 22 April 2013; doi:10.1038/leu.2013.122.

10 Stransky N, Egloff AM, Tward AD, Kostic AD, Cibulskis K, Sivachenko A et al. The mutational landscape of head and neck squamous cell carcinoma. Science 2011; 333: $1157-1160$.

11 Yokoyama T, Okamura S, Asano Y, Kamezaki K, Numata A, Kakumitsu H et al. A novel mutation in the juxtamembrane intracellular sequence of the granulocyte colony-stimulating factor (G-CSF) receptor gene in a patient with severe congenital neutropenia augments GCSF proliferation activity but not through the MAP kinase cascade. Int J Hematol 2005; 82: 28-34.

12 Liu F, Kunter G, Krem MM, Eades WC, Cain JA, Tomasson MH et al. Csf3r mutations in mice confer a strong clonal HSC advantage via activation of Stat5. J Clin Invest 2008; 118: 946-955.

13 Itzykson R, Kosmider O, Renneville A, Morabito M, Preudhomme C, Berthon C et al. Clonal architecture of chronic myelomonocytic leukemias. Blood 2013; 121: 2186-2198.

14 Damm F, Itzykson R, Kosmider O, Droin N, Renneville A, Chesnais V et al. SETBP1 mutations in 658 patients with myelodysplastic syndromes, chronic myelomonocytic leukemia and secondary acute myeloid leukemias. Leukemia 2013; 27: 1401-1403.

15 Itzykson R, Kosmider O, Renneville A, Gelsi-Boyer V, Meggendorfer M, Morabito M et al. Prognostic score including gene mutations in chronic myelomonocytic leukemia. J Clin Oncol 2013; 31: 2428-2436.

\title{
OPEN
}

\section{Prediction of poor outcome in CLL patients following first-line treatment with fludarabine, cyclophosphamide and rituximab}

\section{Leukemia (2013) 27, 1949-1952; doi:10.1038/leu.2013.190}

Chemoimmunotherapies like the combination of fludarabine, cyclophosphamide, and rituximab (FCR) achieve response rates as high as $95 \%$ in patients with previously untreated chronic lymphocytic leukemia (CLL). ${ }^{1}$ Further improvements in clinical outcome will therefore mainly depend on attaining more durable remissions in those responders who suffer an early relapse. In general, progression of disease within 24 months after intensive chemoimmunotherapy is regarded as unsatisfactory ${ }^{2}$ and might be associated with a shortened overall survival $(\mathrm{OS})^{3}{ }^{3}$

We therefore conducted this analysis to identify patients at high risk of early progression after FCR chemoimmunotherapy. The construction of a risk predictor for early relapse utilized the comprehensive set of molecular, genetic, clinical and minimal residual disease (MRD) data that were assessed within the CLL8 trial. This large randomized phase 3 study of the German CLL study group had compared FCR with fludarabine/cyclophosphamide (FC) treatment. Risk features were prospectively determined in CLL8 as described before. ${ }^{1,4}$ In addition, TP53 mutations in exons 2-11 were analyzed by a resequencing chip with confirmatory Sanger sequencing. Risk features in the FCR arm of the CLL8 trial were distributed as expected for a cohort of fit patients requiring firstline treatment (Table 1). MRD quantification was scheduled only for German and Austrian patients per CLL8 protocol. Mainly because of lacking MRD assessments, the patients with sufficient data for risk classification $(n=139)$ were a sub-population of the intention-to-treat $(n=408)$ cohort. However, with the exception of a marginally higher frequency of an unmutated IGHV gene in former cohort $(P=0.02)$, both patient groups did not differ significantly in any of the 14 baseline features assessed. Compared with previous publications of CLL8 results, ${ }^{1,4}$ data were analyzed for this investigation at a more mature median follow-up of 70 months and after 550 patients in the intention-to-treat population $(n=817)$ had progressed or died. Fisher's exact and log-rank tests, respectively, were used to compare proportions of patients and time-dependent variables between patient cohorts. The two sided significance level was set at 0.05 . The construction of the risk score additionally utilized published data on the prognostic significance of TP53 aberrations from other trials. ${ }^{6-9}$

In univariate analyses (Table 1$)$, both high $\left(\geqslant 10^{-2}\right)$ and intermediate $\left(<10^{-2}\right.$ but $\left.\geqslant 10^{-4}\right)$ MRD levels, failure to achieve a complete remission according to iwCLL guidelines, ${ }^{10}$ TP53 abnormalities (that is, TP53 mutations and/or deletions), absence of chromosomal deletion 13q, presence of deletion 11q, unmutated IGHV genes, elevated serum levels of thymidine kinase (s-TK) and $\beta_{2}$-microglobulin $(s-\beta 2 m)$, as well as male gender, were all significantly associated with shorter progression free survival (PFS) after FCR. Cox proportional hazard models were fitted in order to assess the independent prognostic significance of those covariates. After stepwise forward selection using data from 121 patients with information on all risk feature available, both high (hazard rate (HR) 29.8, $P<0.001$ ) and intermediate MRD levels (HR 3.4, $P<0.001$ ), an unmutated IGHV gene (HR 2.5, $P=0.001$ ) and male gender (HR 1.8, $P=0.045$ ) remained significantly associated with short PFS.

We decided to base the novel risk predictor on MRD being the variable most closely associated with short PFS in multivariate analysis. High MRD levels as such described poor risk patients with a median PFS of 11.7 months only, comprising $\sim 13 \%$ of all patients. Patients with intermediate MRD levels $(22 \%$ of all patients) progressed after a median of 38 months, thus including patients with both satisfactory and unsatisfactory outcome. We hypothesized that covariates which besides MRD levels independently predicted PFS in multivariate models (IGHV status, gender) might be able to subdivide this heterogeneous patient group. Patients with unmutated IGHV who attained intermediate MRD levels after FCR experienced a median PFS of 32 months compared with 45 months in patients with mutated IGHV gene (Figure 1a, HR 3.2, $P=0.02$ ). In contrast, gender was less potent to predict PFS in intermediate MRD level patients (Figure $1 \mathrm{~b}$, median PFS, 37 vs 44 months, HR 0.6, $P=0.30$ ). In summary, based on our data from the CLL8 trial, a short PFS after FCR can be expected in patients who either present with high MRD levels $\left(\geqslant 10^{-2}\right)$ after therapy, or in those who attain intermediate MRD levels $\left(<10^{-2}\right.$ but $\geqslant 10^{-4}$ ) and carry an unmutated IGHV gene. 
Table 1. Patient's characteristics and univariate association with PFS

\begin{tabular}{|c|c|c|c|c|c|}
\hline & ITT population $(\mathrm{n}=408)$ & Analysis set $(\mathrm{n}=139)$ & Median PFS (months) & Hazard rate $(95 \% \mathrm{Cl})$ & P-value \\
\hline Male & $74 \%(303 / 408)$ & $78 \%(109 / 139)$ & 54.2 & $1.51(1.12-2.04)$ & 0.007 \\
\hline B-symptoms & $41 \%(167 / 407)$ & $35 \%(49 / 139)$ & 56.0 & $0.92(0.71-1.20)$ & 0.52 \\
\hline Binet $C$ & $31 \%(126 / 407)$ & $32 \%(44 / 139)$ & 42.6 & $1.27(0.97-1.65)$ & 0.08 \\
\hline IGHV unmutated & $64 \%(197 / 310)$ & $71 \%(95 / 134)$ & 41.8 & $2.67(1.92-3.72)$ & $<0.001$ \\
\hline Deletion $(13 q)^{a}$ & $34 \%(105 / 311)$ & $34 \%(44 / 131)$ & 77.5 & $0.50(036-0.70)$ & $<0.001$ \\
\hline Deletion $(17 p)^{a}$ & $7 \%(22 / 311)$ & $5 \%(7 / 131)$ & 11.3 & $3.91(2.39-6.40)$ & $<0.001$ \\
\hline TP53 mutation & $10 \%(31 / 313)$ & $8 \%(11 / 133)$ & 15.4 & $3.17(2.09-4.82)$ & $<0.001$ \\
\hline Deletion (17p) and/or TP53 mutation & $11 \%(34 / 305)$ & $8 \%(11 / 133)$ & 15.4 & $3.32(2.21-4.99)$ & $<0.001$ \\
\hline$s-\beta 2 m \geqslant 3.5 \mathrm{mg} / \mathrm{l}$ & $33 \%(106 / 318)$ & $30 \%(40 / 134)$ & 43.3 & $1.50(1.13-2.00)$ & 0.005 \\
\hline $\mathrm{s}-\mathrm{TK} \geqslant 10 \mathrm{U} / \mathrm{l}$ & $73 \%(232 / 318)$ & $78 \%(105 / 134)$ & 51.9 & $1.70(1.21-2.39)$ & 0.002 \\
\hline MRD level $\geqslant 10^{-4}$ to $<10^{-2}$ & $24 \%(35 / 143)$ & $22 \%(31 / 139)$ & 38.4 & $2.8(1.8-4.6)^{\mathrm{b}}$ & $<0.001$ \\
\hline MRD level $\geqslant 10^{-2}$ & $13 \%(18 / 143)$ & $13 \%(18 / 139)$ & 11.7 & $20.0(10.4-38.6)^{\mathrm{b}}$ & $<0.001$ \\
\hline
\end{tabular}

Abbreviations: $\mathrm{Cl}$, confidence interval; $\mathrm{CLL}$, chronic lymphocytic leukemia; $\mathrm{CR}$, complete remission; ECOG, Eastern Cooperative Oncology Group; $\mathrm{FCR}$, fludarabine, cyclophosphamide, and rituximab; ITT, intention-to-treat; MRD, minimal residual disease; PFS, progression free survival; s- $\beta 2 \mathrm{~m}$, $\beta_{2}$-microglobulin; s-TK, serum levels of thymidine kinase. Baseline characteristics and risks features of the CLL8 trial patients who received FCR are given for all patients in the intention-to-treat (ITT) population and in the subgroup of patients with sufficient data for risk classification according to the algorithm. Median PFS, hazard rates and significance of the association to PFS in univariate analysis are tabulated per risk feature. Hazard rates are computed in comparison with all other patients with available data on a particular risk feature, unless otherwise stated. MRD data are assessed in peripheral blood, 2 months after completion of therapy. ${ }^{\mathrm{a}}$ Chromosomal aberrations were assessed according to the Döhner hierarchical model. ${ }^{15}{ }^{\mathrm{b}} \mathrm{Compared}$ with MRD levels $<10^{-4}$ (MRD negativity according to iwCLL definition ${ }^{10}$ ).

TP53 abnormalities were besides high MRD levels, the risk features most strongly associated with poor outcome (median PFS 15 months) in univariate analysis (Table 1), warranting detailed analyses of this variable. There was a considerable but incomplete overlap between those two disadvantaged patient groups. Six out of the total 11 patients with TP53 abnormalities had high MRD levels of $\geqslant 10^{-2}$ (median PFS 11 months, Supplementary Figure 2a), whereas three patients achieved intermediate MRD levels (median PFS 30 months). Two patients with either a TP53 mutation or deletion achieved an MRD-negative response (MRD levels $\left.<10^{-4}\right)$. Thereof, one patient progressed after 54 months and the second one was censored at 69 months without progression. Patients who presented with intermediate MRD and carried a TP53 aberration showed a trend for shorter median PFS compared with patients without TP53 aberrations who achieved the same MRD levels ( 30 vs 40 months, $P=0.42$, Supplementary Figure 2a). The lack of significance likely reflects low patient numbers. These observations suggested that the risk of progression in patients with TP53 abnormalities differs by MRD status and that TP53 status might impact on median PFS in patients with intermediate MRD levels. The prognostic significance of MRD within the subgroup of patients with TP53 aberrations was recently also reported by the UK CLL206 investigators. ${ }^{6}$ Nevertheless, compared with published data on patients without TP53 aberrations, ${ }^{7}$ even MRD-negative patients with this genetic lesion experienced a relatively short PFS in that trial. This suggests that both TP53 aberrations and MRD response independently predict for PFS in CLL. All patients with a TP53 aberration in our analysis of CLL8 data carried an unmutated IGHV gene. However, previous reports had shown that $\sim 25 \%$ of all patients with TP53 aberrations expressed mutated IGHV genes. ${ }^{7-9}$ The substantial proportion of patients carrying either one of the two risk features only, the well-reported adverse impact of TP53 aberrations, and the already existing iwCLL recommendation to assess this lesion before therapy motivated us to incorporate that feature into the risk score.

In summary, we characterized patients at high risk for early progression as presenting with high MRD levels after therapy or attaining intermediate MRD levels plus carrying at least one of the adverse prognostic markers TP53 aberrations or unmutated IGHV status. Low risk for early progression was predicted by low MRD levels $\left(<10^{-4}\right)$ irrespective of any additional feature or intermediate MRD levels in patients who carried neither unmutated IGHV genes nor a TP53 aberration. Median PFS of the high-risk cohort that comprised $\sim 29 \%$ of all patients was 22 months compared with 68 months for low-risk patients (HR 6.6, $P<0.0001$, Figure 1c). High-risk patients had a median OS of 64 months only, whereas median OS was not reached in the low-risk group (HR 4.4, $P<0.0001$, Figure 1d).

We next investigated the reliability of the proposed algorithm applying it to patients treated in the FC arm of the CLL8 trial (Supplementary Table 1). High-risk patients after FC achieved a median PFS of 29 months compared with 64 months for the lowrisk patients (HR $4.2195 \% \mathrm{Cl} 2.75-6.45 ; P<0.001$, Supplementary Figure 1c) and an OS of 77 vs 90 months (HR 2.20 95\% Cl 1.20-4.03; $P=0.01$, Supplementary Figure 1d). These results suggest that the algorithm identifies poor risk patients not only after FCR, but similarly after FC treatment. Moreover, in an independent multivariate analysis $(n=115), \mathrm{MRD}$ again appeared as highly associated with PFS (high MRD levels, HR 18.8, $P<0.005$; intermediate MRD levels, HR 2.2, $P=0.005$ ). Additional prognostic markers with independent impact on PFS were IGHV status (HR 1.8, $P=0.02$ ) and TP53 aberrations (HR 2.9; $P=0.002$ ), thus corroborating the choice of variables for the risk predictor.

While prognostic indices for time to first treatment and $\mathrm{OS}^{11,12}$ have been proposed before, only limited information exists to predict PFS after treatment. To the best of our knowledge, there is only one previous analysis from a randomized trial that established a multivariable predictor for the likelihood of progression after treatment. ${ }^{13}$ The poor risk category of that model was confined to patients with a TP53 deletion (6\% of all patients), whereas the poor risk group identified by our MRD based model comprises a substantially larger patient group ( $29 \%$ of all patients).

Allogeneic stem cell transplantation remains the treatment of choice for the minority of eligible ultra-high-risk patients who carry a TP53 aberration. ${ }^{14}$ The majority of high-risk patients identified by the novel algorithm, however, does not carry a TP53 aberration or might be ineligible for transplantation. We consider 
a

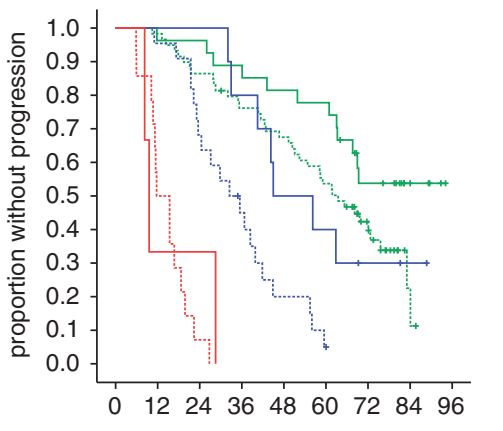

b

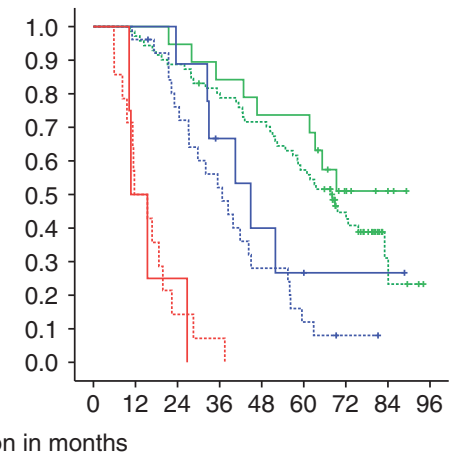

C

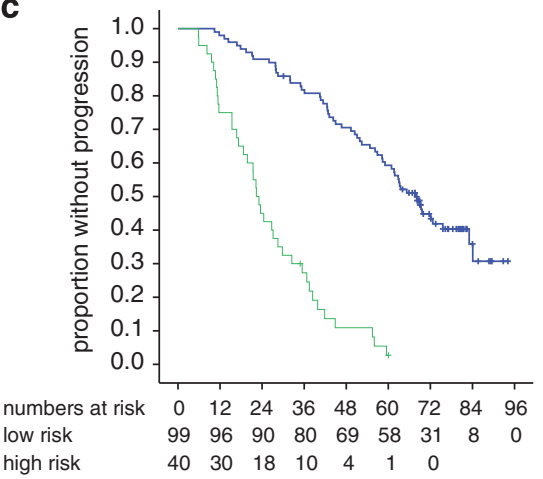

d

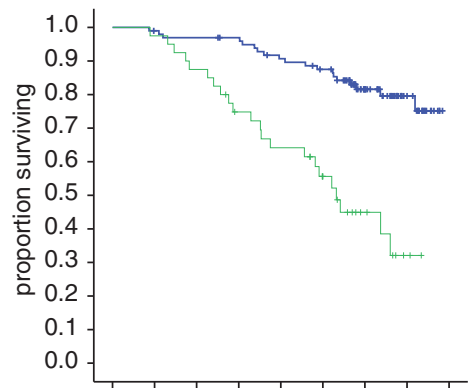

$\begin{array}{llllllllll}\text { numbers at risk } & 0 & 12 & 24 & 36 & 48 & 60 & 72 & 84 & 96\end{array}$

Low risk $99 \begin{array}{lllllllll}97 & 95 & 93 & 86 & 81 & 50 & 20 & 0\end{array}$ High risk $\begin{array}{lllllllll}40 & 39 & 35 & 28 & 24 & 17 & 8 & 2 & 0\end{array}$

Time since randomization in months

Figure 1. Combination of individual risk features to predict PFS and OS after FCR treatment. MRD levels are grouped as high (red curves, $\geqslant 10^{-2}$ ), intermediate (blue curves, $\geqslant 10^{-4}$ to $<10^{-2}$ ) and low (green curves, $<10^{-4}$ ) in $(\mathbf{a}, \mathbf{b})$. Patients classified as high and low risk by the algorithm are symbolized using green and blue curves, respectively, in (c, d). (a) PFS by MRD levels and IGHV status ( $n=135$ ). For patients who attained intermediate MRD levels, a mutated IGHV (solid lines) was associated with longer PFS compared with patients with unmutated IGHV (dashed lines) status (HR 3.2, $P=0.02, n=32$ ). IGHV had a lesser impact on PFS in high-level MRD (HR 1.6, $P=0.56, n=17)$ and low-level MRD patients (HR 1.9, $P=0.046, n=86$ ). (b) PFS by MRD levels in female (solid curves) and male (dashed curves) patients (total $n=143$ ), showing a marginally, but not significantly better outcome in women who achieved intermediate $(n=35, \mathrm{HR} 0.62 P=0.30$,) and low-level MRD ( $n=90$, HR $0.69, P=0.29$ ), while no difference at all was detectable in high-level MRD patients $(n=18, \mathrm{HR} 1.2, P=0.8)$. (c) PFS in patients classified as high and low risk by the algorithm (HR 6.6, $P<0.001$ ). (d) OS in patients classified as high and low risk by the algorithm (HR 4.4, $P<0.001$ ).

such patients at high risk of early progression ideal candidates for clinical trials testing the efficacy of novel maintenance strategies in this disadvantaged patient group.

\section{CONFLICT OF INTEREST}

Employment or leadership in F. Hoffmann-La Roche: MW, GFR; Consultant or advisory role in F. Hoffmann-La Roche: SS, CW, MK, MH; Stock Ownership in F. Hoffmann-La Roche: MW; Honoraria from F. Hoffmann-La Roche: SB, SS, TZ, DW, AB, BE, CW, PS, GH, $\mathrm{MK}, \mathrm{MH}$; Research funding from Hoffmann-La Roche: SB, MR, SS, CW, BE, MK, MH; Other remuneration (travel grants) from F. Hoffmann-La Roche: AMF, KF, PS, ; RB, DW, MHe and NP declare no conflict of interest.

\section{ACKNOWLEDGEMENTS}

We are grateful to the patients who participated in the CLL8 trial and the physicians who treated them. We also thank Myriam Mendila, Stephan Zurfluh and Jamie Wingate for their excellent support during the conduct of this trial. We wish to thank Elke Harbst, Jamileh Hanani, Maike Starken, Lada Henseleit and Linda Falck for excellent technical assistance, as well as Anne Westermann for data handling. This work was supported by German Jose Carreras Leukemia Foundation (project R 06/03v).

AM Fink ${ }^{1,14}$, S Böttcher ${ }^{2,14}$, M Ritgen ${ }^{2}$, K Fischer $^{1}$, N Pflug ${ }^{1}$,

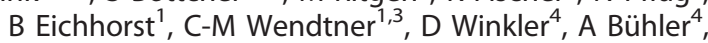
T Zenz ${ }^{4,5,6}$, P Staib ${ }^{7}, \mathrm{~J} \mathrm{Mayer}^{8}, \mathrm{M} \mathrm{Hensel}^{5,6,9}, \mathrm{G} \mathrm{Hopfinger}^{10,11^{\prime}}$, M Wenger ${ }^{12}$, G Fingerle-Rowson ${ }^{1,12}, \mathrm{H}^{2}$ Döhner $^{4}, \mathrm{M} \mathrm{Kneba}^{2}$, S Stilgenbauer ${ }^{4}$, R Busch $^{13}$ and M Hallek ${ }^{1}$

${ }^{1}$ Department I of Internal Medicine and Center of Integrated Oncology Cologne Bonn, Cologne, Germany;
${ }^{2}$ Second Department of Medicine, University Hospital of Schleswig-Holstein, Campus Kiel, Kiel, Germany;

${ }^{3}$ Department I of Internal Medicine, Klinikum München-Schwabing, Munich, Germany; ${ }^{4}$ Department of Internal Medicine III, University of Ulm, Ulm, Germany;

${ }^{5}$ Department of Translational Oncology, National Center for Tumor Diseases (NCT) and German Cancer Research Center (DKFZ), Heidelberg, Germany;

${ }^{6}$ Department of Medicine V, University of Heidelberg, Heidelberg, Germany;

${ }^{7}$ Klinik für Hämatologie und Onkologie, St. Antonius Hospital Eschweiler, Eschweiler, Germany;

${ }^{8}$ Department of Internal Medicine, Hematology and Oncology, University Hospital Brno, and CEITEC, Masaryk University, Brno, Czech Republic;

${ }^{9}$ Mannheimer Onkologie Praxis, Mannheim, Germany; ${ }^{10}$ Department of Medicine III, Hanusch Hospital Vienna, Vienna, Austria;

${ }^{11} 3$ rd Medical Department with Haematology, Medical Oncology, Haemostasiology, Infectious Diseases, and Rheumatology, Paracelsus Medical University, Salzburg, Austria;

${ }^{12}$ Pharmaceutical Division/Global Drug Dev., F. Hoffmann-La Roche Ltd., Basel, Switzerland and

${ }^{13}$ Institute for Medical Statistics and Epidemiology, Technical University, Munich, Germany

E-mail: anna-maria.fink@uk-koeln.de

${ }^{14}$ These authors contributed equally to this work and are therefore joint first authors of this study. 
Preliminary results were presented in part at the Annual meeting of the American Society of Hematology, San Diego, CA, USA, December 8-13, 2011.

\section{REFERENCES}

1 Hallek M, Fischer K, Fingerle-Rowson G, Fink AM, Busch R, Mayer J et al. Addition of rituximab to fludarabine and cyclophosphamide in patients with chronic lymphocytic leukaemia: a randomised, open-label, phase 3 trial. Lancet 2010; 376: 1164-1174.

2 Zenz T, Gribben JG, Hallek M, Dohner H, Keating MJ, Stilgenbauer S. Risk categories and refractory CLL in the era of chemoimmunotherapy. Blood 2012; 119: 4101-4107.

3 Stilgenbauer S, Zenz T. Understanding and managing ultra high-risk chronic lymphocytic leukemia. Hematology Am Soc Hematol Educ Program 2010; 2010 481-488.

4 Bottcher S, Ritgen M, Fischer K, Stilgenbauer S, Busch R, Fingerle-Rowson G et al. Minimal residual disease quantification is an independent predictor of progression free and overall survival in chronic lymphocytic leukemia. A multivariate analysis from the randomized GCLLSG CLL8 trial. J Clin Oncol 2012; 30: 980-988.

5 Bottcher S, Stilgenbauer S, Busch R, Bruggemann M, Raff T, Pott $C$ et al Standardized MRD flow and ASO IGH RQ-PCR for MRD quantification in CLL patients after rituximab-containing immunochemotherapy: a comparative analysis. Leukemia 2009; 23: 2007-2017.

6 Pettitt AR, Jackson R, Carruthers S, Dodd J, Dodd S, Oates M et al. Alemtuzumab in combination with methylprednisolone is a highly effective induction regimen for patients with chronic lymphocytic leukemia and deletion of TP53: final results of the national cancer research institute CLL206 trial. J Clin Oncol 2012; 30: 1647-1655.

7 Zenz T, Eichhorst B, Busch R, Denzel T, Habe S, Winkler D et al. TP53 mutation and survival in chronic lymphocytic leukemia. J Clin Oncol 2010; 28: 4473-4479.
8 Gonzalez D, Martinez P, Wade R, Hockley S, Oscier D, Matutes E et al. Mutational status of the TP53 gene as a predictor of response and survival in patients with chronic lymphocytic leukemia: results from the LRF CLL4 trial. J Clin Oncol 2011; 29: $2223-2229$.

9 Dicker F, Herholz H, Schnittger S, Nakao A, Patten N, Wu L et al. The detection of TP53 mutations in chronic lymphocytic leukemia independently predicts rapid disease progression and is highly correlated with a complex aberrant karyotype. Leukemia 2009; 23: 117-124.

10 Hallek M, Cheson BD, Catovsky D, Caligaris-Cappio F, Dighiero G, Dohner H et al. Guidelines for the diagnosis and treatment of chronic lymphocytic leukemia: a report from the International Workshop on Chronic Lymphocytic Leukemia updating the National Cancer Institute-Working Group 1996 guidelines. Blood 2008; 111: 5446-5456.

11 Wierda WG, O'Brien S, Wang X, Faderl S, Ferrajoli A, Do KA et al. Characteristics associated with important clinical end points in patients with chronic lymphocytic leukemia at initial treatment. J Clin Oncol 2009; 27: 1637-1643.

12 Shanafelt TD, Jenkins G, Call TG, Zent CS, Slager S, Bowen DA et al. Validation of a new prognostic index for patients with chronic lymphocytic leukemia. Cancer 2009; 115: 363-372.

13 Oscier D, Wade R, Davis Z, Morilla A, Best G, Richards S et al. Prognostic factors identified three risk groups in the LRF CLL4 trial, independent of treatment allocation. Haematologica 2010; 95: 1705-1712.

14 Dreger P, Corradini P, Kimby E, Michallet M, Milligan D, Scheteliget al. Indications for allogeneic stem cell transplantation in chronic lymphocytic leukemia: the EBMT transplant consensus. Leukemia 2007; 21: 12-17.

15 Dohner H, Stilgenbauer S, Benner A, Leupolt E, Krober A, Bullinger L et al. Genomic aberrations and survival in chronic lymphocytic leukemia. $N$ Engl J Med 2000; 343: 1910-1916.

(c) (1) $\Theta$ This work is licensed under a Creative Commons AttributionNonCommercial-NoDerivs 3.0 Unported License. To view a copy of this license, visit http://creativecommons.org/licenses/by-nc-nd/3.0/

Supplementary Information accompanies this paper on the Leukemia website (http://www.nature.com/leu) 